\title{
P53 in acute respiratory distress syndrome
}

\author{
Nektarios Barabutis ${ }^{1} \mathbb{D}$
}

Received: 4 June 2020 / Revised: 29 July 2020 / Accepted: 21 August 2020 / Published online: 4 September 2020

(c) Springer Nature Switzerland AG 2020

\begin{abstract}
P53 is a tumor suppressor protein, associated with strong anti-inflammatory activities. Recent evidence suggest that this transcription factor counteracts lung inflammatory diseases, including the lethal acute respiratory distress syndrome. Herein we provide a brief discussion on the relevant topic.
\end{abstract}

Keywords Inflammation $\cdot$ Acute lung injury $\cdot$ Unfolded protein response $\cdot$ Heat shock proteins

The severities of the acute respiratory distress syndrome (ARDS), including the ARDS related to COVID19 , are associated with thousands of deaths worldwide. The current therapeutic approaches do not suffice to reduce the unacceptably high mortality rates of that lethal disorder. Hence, intense efforts are oriented towards the development of efficient medical countermeasures. Since lung endothelial hyper-permeability due to the "cytokine storm" is both a cause and consequence of ARDS, the elucidation of the mechanisms involved in the regulation of the lung endothelium is of the utmost need. Delineation of the molecular signaling governing the vascular barrier function may deliver new and exciting therapeutic possibilities for those patients in need [1].

P53 is an anti-inflammatory transcription factor, which devises cellular responses against a diverse variety of environmental stimuli. Indeed, it is subjected to a reciprocal regulation with the nuclear factor-kappa B (NF- $\mathrm{kB})$. The exact nature of those interrelations has been recently described in a recent exceptional study by Carra et al. [2]. The proficient investigators explored the great depths of the tumoral P53/ $\mathrm{NF}-\kappa \mathrm{B}$ network and released their own perspectives in the corresponding field [2].

P53 counteracts stress by promoting cell death, senescence, or cell cycle arrest by utilizing oxidative phosphorylation. $\mathrm{NF}-\kappa \mathrm{B}$ initiates immune responses by aerobic

Nektarios Barabutis

barabutis@ulm.edu

1 School of Basic Pharmaceutical and Toxicological Sciences, College of Pharmacy, University of Louisiana Monroe, 1800 Bienville Drive, Monroe, LA 71201, USA glycolysis [3]. The activation of one of those proteins results in the deactivation of the other, hence inhibition of NF- $\mathrm{kB}$ results in the activation of P53 functions [4]. Chronic infections by mycoplasma as well as NF- $\mathrm{\kappa B}$-activating peptides reduce $\mathrm{P} 53$, contributing to the progression of malignant transformations [5]. P53 is required for the repression of NF- $\kappa \mathrm{B}$ by the glucocorticoid receptor [6], and P53 deficiency in mice was shown to potentiate the LPS-induced inflammation [7]. Moreover, P53 inhibits inflammation by antagonizing NF- $\mathrm{BB}[8]$.

The anti-inflammatory activities of P53 in human pathophysiology are not limited to cancer tissues. Indeed, it appears that this transcription factor exerts the capacity to protect against the LPS-induced lung endothelial hyperpermeability, by suppressing the inflammatory RhoA/MLC2 pathway, thus reducing the formation of the F-actin fibers [9]. Further studies revealed that P53 enhances endothelial barrier function by suppressing the actin-severing activity of cofilin, as well as by mediating the opposing activities of Rac1/RhoA both in vitro and in mutant mice overexpressing P53 [10].

Cofilin is the downstream target of the Rac1/p21-activated kinase/LIMK axis [11, 12], and has been associated with hyper-permeability responses [13]. Activation of this molecular cascade by P53 inducers (i.e. Hsp90 inhibitors) phosphorylates, hence deactivates, cofilin [10]. On the other hand, this endothelial defender (P53) [14] suppresses the RhoA pathway via p190RhoGAP induction. This is a Rho family GTPase-activating protein which regulates actin stress fiber dynamics via hydrolysis of Rho-GTP, and it is a suppressor of the RhoA/MLC2 pathway [15]. 
The phosphorylation of P53 is modulated by both LPS and Hsp90 inhibitors, indicating that activated (phospho) P53 is involved in lung hyper-permeability responses [16]. Furthermore, studies in bovine lung microvascular cells indicated that the protective effects of P53 are associated with the suppression of the inflammatory apyrimidinic endonuclease 1/redox factor-1 (APE1/Ref1) [17]; and the reduction of the reactive oxygen species [18]. APE1/Ref-1 is a redox signalling factor, which regulates the DNA binding activities of NF-KB and hypoxia-inducible transcription factor $1 \alpha$ [19]. Thus, it exerts a major role in promoting inflammation and serves as a therapeutic target in human disease [20].

Interestingly, this endothelium defender (P53) [14] has been shown to be a direct target of the unfolded protein response (UPR) [21, 22]. UPR acts to restore impaired functions of damaged tissues [23], including the lungs [24, 25] and affects P53 levels in a positive manner. In lung bovine cells the UPR inductors Brefeldin A, dithiothreitol, and thapsigargin induced P53, while the UPR inhibitors N-acetyl cysteine, kifunensine, and ATP-competitive IRE1 $\alpha$ kinaseinhibiting RNase attenuator produced the opposite effects. Hence P53 may mediate, or even initiate, UPR-mediated responses towards the maintenance of lung homeostasis [26].

This multifaceted maestro (P53) is strongly involved in the protective effects of Hsp90 inhibitors and GHRH antagonists in the lung microvasculature [27, 28]. Those anti-cancer and anti-inflammatory agents induce UPR both in vivo and in vitro. Hence, the existence of a new molecular network in the context of P53/UPR/Hsp90 [29], which operates towards the repair of the severely inflamed lungs, is highly possible $[30,31]$. Pharmacological "fine-tuning" of that meticulously orchestrated network, may deliver novel ways to restore respiratory functions in the hospitalized ARDS patients.

Funding Our research is supported by the R\&D, Research Competitiveness Subprogram (RCS) of the Louisiana Board of Regents through the Board of Regents Support Fund (LEQSF(2019-22)-RD-A-26) (P.I: NB), the FRSP award of the College of Pharmacy (ULM) (P.I:NB), and the National Institute of General Medical Sciences of the National Institutes of Health (5 P20 GM103424-15, 3 P20 GM103424-15S1).

\section{References}

1. Barabutis $\mathrm{N}$ (2019) Unfolded protein response in acute respiratory distress syndrome. Lung 197(6):827-828

2. Carra G, Lingua MF, Maffeo B, Taulli R, Morotti A (2020) P53 vs $\mathrm{NF}-\kappa \mathrm{B}$ : the role of nuclear factor-kappa $\mathrm{B}$ in the regulation of $\mathrm{p} 53$ activity and vice versa. Cell Mol Life Sci. https://doi.org/10.1007/ s00018-020-03524-9
3. Ak P, Levine AJ (2010) p53 and NF-kappaB: different strategies for responding to stress lead to a functional antagonism. FASEB J 24(10):3643-3652

4. Gurova KV et al (2005) Small molecules that reactivate p53 in renal cell carcinoma reveal a NF-kappaB-dependent mechanism of p53 suppression in tumors. Proc Natl Acad Sci USA 102(48):17448-17453

5. Gudkov AV, Komarova EA (2016) p53 and the carcinogenicity of chronic inflammation. Cold Spring Harb Perspect Med 6(11):a026161

6. Murphy SH et al (2011) Tumor suppressor protein (p)53, is a regulator of NF-kappaB repression by the glucocorticoid receptor. Proc Natl Acad Sci USA 108(41):17117-17122

7. Uddin MA et al (2020) P53 deficiency potentiates LPS-induced acute lung injury in vivo. Curr Res Physiol 3:30-33

8. Komarova EA et al (2005) p53 is a suppressor of inflammatory response in mice. FASEB J 19(8):1030-1032

9. Barabutis $\mathrm{N}$ et al (2015) p53 protects against LPS-induced lung endothelial barrier dysfunction. Am J Physiol Lung Cell Mol Physiol 308(8):L776-L787

10. Barabutis $\mathrm{N}$ et al (2018) Wild-type p53 enhances endothelial barrier function by mediating RAC1 signalling and RhoA inhibition. J Cell Mol Med 22(3):1792-1804

11. Slee JB, Lowe-Krentz LJ (2013) Actin realignment and cofilin regulation are essential for barrier integrity during shear stress. J Cell Biochem 114(4):782-795

12. Bernstein BW, Bamburg JR (2010) ADF/cofilin: a functional node in cell biology. Trends Cell Biol 20(4):187-195

13. Bravo-Cordero JJ et al (2013) Functions of cofilin in cell locomotion and invasion. Nat Rev Mol Cell Biol 14(7):405-415

14. Uddin MA, Barabutis N (2019) P53: the endothelium defender. J Cell Biochem 120:10952

15. Arthur WT, Burridge K (2001) RhoA inactivation by p190RhoGAP regulates cell spreading and migration by promoting membrane protrusion and polarity. Mol Biol Cell 12(9):2711-2720

16. Barabutis N, Uddin MA, Catravas JD (2019) Hsp90 inhibitors suppress P53 phosphorylation in LPS-induced endothelial inflammation. Cytokine 113:427-432

17. Uddin MA et al (2019) P53 supports endothelial barrier function via APE1/Ref1 suppression. Immunobiology 224(4):532-538

18. Akhter MS, Uddin MA, Barabutis N (2020) P53 regulates the redox status of lung endothelial cells. Inflammation 43(2):686-691

19. Jedinak A et al (2011) Apurinic/apyrimidinic endonuclease 1 regulates inflammatory response in macrophages. Anticancer Res 31(2):379-385

20. Thakur S et al (2014) APE1/Ref-1 as an emerging therapeutic target for various human diseases: phytochemical modulation of its functions. Exp Mol Med 46:e106

21. Byun S, Namba T, Lee SW (2015) Losing p53 loosens up ERstress. Aging (Albany NY) 7(11):895-896

22. Fusee LTS et al (2020) Alternative mechanisms of p53 action during the unfolded protein response. Cancers (Basel) 12(2):401

23. Kubra KT et al (2020) Unfolded protein response in cardiovascular disease. Cell Signal 73:109699

24. Barabutis N (2020) Unfolded protein response in lung health and disease. Front Med 7:344

25. Hetz C, Axten JM, Patterson JB (2019) Pharmacological targeting of the unfolded protein response for disease intervention. Nat Chem Biol 15(8):764-775

26. Akhter MS, Uddin MA, Barabutis N (2019) Unfolded protein response regulates $\mathrm{P} 53$ expression in the pulmonary endothelium. J Biochem Mol Toxicol 33(10):e22380

27. Barabutis $N(2020)$ Heat shock protein 90 inhibition in the inflamed lungs. Cell Stress Chaperones 25(2):195-197 
28. Uddin MA et al (2019) GHRH antagonists support lung endothelial barrier function. Tissue Barriers 7(4):1669989

29. Barabutis N, Siejka A (2020) The highly interrelated GHRH, p53, and Hsp90 universe. Cell Biol Int 44:1558

30. Kubra KT et al (2020) Hsp90 inhibitors induce the unfolded protein response in bovine and mice lung cells. Cell Signal 67:109500

31. Barabutis N (2020) Growth hormone releasing hormone in the unfolded protein response context. Endocrine 67(2):291-293
Publisher's Note Springer Nature remains neutral with regard to jurisdictional claims in published maps and institutional affiliations. 